\title{
Evalu-light: A practical approach for evaluating character lighting in real-time
}

\author{
Pisut Wisessing \\ Trinity College Dublin \\ pisut.wisessing@tcd.ie
}

\author{
Rachel McDonnell \\ Trinity College Dublin \\ rachel.mcdonnell@tcd.ie
}


Figure 1: Screenshots showing the Digital Lighting Tool A being used in the Speed \& Accuracy Task (left) and the Perceptuallybased Lighting Tool B being used in the Appeal Task (right).

\begin{abstract}
The demand for cartoon animation content is on the rise, driven largely by social media, video conferencing and virtual reality. New content creation tools and the availability of open-source game engines allow characters to be animated by even novice creators (e.g., Loomie and Pinscreen for creating characters, Hyprface and MotionX for facial motion capture, etc,). However, lighting of animated characters is a skilled art-form, and there is a lack of guidelines for lighting characters expressing emotion in an appealing manner.

Recent perceptual research has attempted to provide user guidelines through rigorous and labor-intensive rating-scale experiments exploring many parameters of light [Wisessing et al. 2016, 2020]. We propose an alternative approach based on the method of adjustment, similar to common lighting tools in $3 \mathrm{D}$ content creation software, but with the power of modern real-time graphics. Our framework allows users to interactively adjust lighting parameters and instantly assess the results on the animated characters, instead of having to wait for the render to complete.
\end{abstract}

\section{CCS CONCEPTS}

- Computing methodologies $\rightarrow$ Computer graphics; Perception; Animation; Visibility.

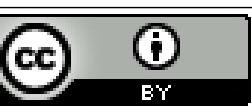

This work is licensed under a Creative Commons Attribution International 4.0 License.

SAP '20, September 12-13, 2020, Virtual Event, USA

(C) 2020 Copyright held by the owner/author(s)

ACM ISBN 978-1-4503-7618-1/20/09.

https://doi.org/10.1145/3385955.3407937

\section{KEYWORDS}

character lighting, virtual characters, evaluation, emotion, appeal

ACM Reference Format:

Pisut Wisessing and Rachel McDonnell. 2020. Evalu-light: A practical approach for evaluating character lighting in real-time. In ACM Symposium on Applied Perception 2020 (SAP '20), September 12-13, 2020, Virtual Event, USA. ACM, New York, NY, USA, 6 pages. https://doi.org/10.1145/3385955.3407937

\section{INTRODUCTION}

There are thousands of animation studios with diverse budgets and expertise worldwide producing content for movies, advertising, TV, games, etc. Additionally, animation content is increasingly being generated by technical developers and non-professionals for virtualassistants, apps, social-media and VR/AR.

Character lighting is one aspect of content creation that is particularly important for establishing the look and feel of a character [Lowell 1992]. However, mastering lighting design to deliver an impactful message require extensive training. Recent perceptual research has attempted to provide user guidelines for appealing illumination through rigorous and labour-intensive experiments exploring many parameters of light (e.g., brightness, shadow, direction, etc.) [Wisessing et al. 2016, 2020] Most previous methods have used rating scales for participants to rate large numbers of pre-generated images (method of constant stimuli) on appeal or realism, etc. Nowadays, since high-quality rendering effects (e.g., soft shadows and subsurface scattering) can be easily achieved in realtime game engines, we propose to leverage these advancements in order to gather data on the perception of lighting for animated characters. Our framework is based on the method of adjustment, similar to common lighting tools in 3D content creation software, but with the power of real-time graphics. Our system allows users 
to interactively adjust lighting parameters and instantly view the results on the animated characters, instead of having to wait for the render to complete.

We show that using our framework can help to speed-up experiment duration, allowing the experimenter to investigate more dependant variables. We focus specifically on brightness and shadow for this experiment, in order to validate our results against previous work. However, our framework can easily be used to analyze any other lighting effects such as render style, lighting color, direction, etc.

We chose to test happiness and sadness because the two emotions have been proved, from linguistics, anthropology to psychology standpoints [Barchard et al. 2017; Landau et al. 2010; Meier and Robinson 2005; Meier et al. 2004; Xu and Labroo 2014], to be directly linked with brightness and shadows, especially by the recent extensive psychophysics experiments conducted in [Wisessing et al. 2020].

Our experiment is the first attempt, to our knowledge, at demonstrating the efficiency of method of adjustments for interactive character lighting, by leveraging the power of real-time graphics. We will make our perceptual tool available for other researchers to investigate lighting parameters, with the hope of accelerating the advancement of research in this field.

\section{RELATED WORK}

In Disney Animation: The Illusion of Life, Thomas and Johnston [1995] explained that appeal, one of the twelve basic principles of animation refers to "anything that a person likes to see," rather than just being good looking One major issue when studying perception of character appeal is the enormous number of variables, such as shape, material, render style, brightness and shadow, just to name a few. Previous studies have investigated only a subset at a time, such as render style [McDonnell et al. 2012; Volante et al. 2016], geometry and material [Zell et al. 2015], stylization level [Wallraven et al. 2007], geometric detail [MacDorman et al. 2009], body shape [Fleming et al. 2016], etc. More specifically, recent work has investigated the role of lighting on character appeal with respect to key-light brightness, key-to-fill ratio and lighting direction [Wisessing et al. 2016, 2020,2019]. They chose to focus their studies on the perceived brightness and shadow amount in the three-point lighting design which has become ubiquitous in portraiture from the painting in the Renaissance to the everyday news broadcasting. Crafting high quality animation and renderings of a character demands time and effort. Investigating a large range of parameters also extends the length of experiments exponentially. As a result, Wisessing et al.'s studies only selected a manageable subset of 9 to 16 data points that evenly represent the entire two-dimensional perceptual space of character lighting described in [Wisessing et al. 2019], which are considerably too sparse for practical applications despite their conclusive findings.

As part of their work on perception of appeal, Wisessing et al. [2020] also investigated emotion intensity and the relationship with lighting parameters. Additionally, a study by Barchard et al. [2017] investigated cross-cultural figurative language and emotions and confirmed that the dichotomous descriptor "bright" is associated with happiness and "dark" is associated with sadness implying that there is a semantic association between emotions and brightness. In addition, Xu and Labroo [2014] found that bright light increases people's perception of heat, which in turn activates their hot emotional system, leading to intensified affective reactionspositive and negative-to different kinds of stimuli. In this work, we investigate the "if", given the task of manipulating light in real-time, participants would use the cultural references to a greater degree than when asked to judge perception of emotion on a range of characters presented at different levels of brightness and shadow intensity.

There are a number of different methodologies for presenting stimuli in behavioural experiments. For example, the method of constant stimuli is where stimuli are chosen at suitably located points in the domain and are then repeatedly presented to the participant in a random order, along with a reference stimulus [Cunningham and Wallraven 2011]. The method of adjustment is the most direct method, where a range of stimulus intensities are available and the participants task is to move the stimulus to their threshold (e.g., [Fechner et al. 1966; Grondin 2016]). A recent survey of research on the perception of virtual characters [Zell et al. 2019] stated that despite the number of publications on the topic, we are still far from fully understanding the perception of virtual characters. Additionally, they state that the downside of current approaches using the method of constant stimuli is the amount of time required and the fact that testing combinations of parameters grows exponentially. In this paper, we investigate leveraging modern game-engine capabilities to speed-up the investigation of rendering parameters.

Interactive parameter adjustments are common in various image manipulation tasks such as tweaking brightness and contrast for photo enhancement [Koyama et al. 2017] or altering the parameters of tone mapping operators [Yoshida et al. 2006]. However, it has not been feasible for experiments that require real-time renders of a 3D scene with advanced features such as sub-surface scattering and soft shadows until the development of modern graphics hardware and game engines.

\section{STIMULI CREATION}

For the CG model, we acquired the animated Mery ${ }^{1}$ character in Alembic ${ }^{2}$ file format from [Wisessing et al. 2020] available for download at their website, and we closely followed the steps described in their study to recreate similar materials and lighting, so that we could compare our result to theirs.

The animated Alembic caches of Mery displaying neutral expression, happiness, and sadness were imported to Unreal Engine version 4.21 where the materials and the lighting were setup. Threepoint lighting used in this study is a well-known illumination design portrait for photography and film, consisting of a key light-the primary source illuminating the character, a fill light brightening up the shadow side of the character, and a rim light separating the character from the background. The three lights are typically area lights, but in our experiments, they were replaced by grids of point lights for efficiency while retaining comparable shadow quality. The camera was placed directly in front of the character at

\footnotetext{
${ }^{1}$ http://www.meryproject.com

${ }^{2}$ https://www.alembic.io/
} 
eye-level and only the face, neck and shoulders of the characters were visible.

During the experiment, the animation of the character was looped. The brightness of the key and fill lights were controlled by the participant and the changes were rendered in real-time.

\section{DIGITAL LIGHTING TOOLS}

We tested two lighting tools, one with the traditional continuous adjustment (Tool A) and the other with the power-of-two adjustment (Tool B). The latter one is equivalent to the conventional unit of stop, commonly used in cinematography and photography (a stop up is doubling and a stop down is halving a light quantity.) Wisessing et al. [2019] confirmed the doubling and halving to be the natural perceived interval where the human visual system can discriminate brightness and shadows. Wisessing et al. [2020] later adopted the-power-of-two interval to create their constant stimuli. Moreover, Tool B was designed specifically to test if limiting the selections to a small subset of data points evenly sampled from the perceived lighting space [Wisessing et al. 2019] can further speed-up the experiment process.

Our experiments were created and run on the Unreal Engine 4 platform. The participants were asked to control the lighting of the scene, rendered in the middle of the screen, using one of the two provided tools, A or B, located on the right side of the screen (Figure 1).

Tool A had two independent control sliders (Figure 1 left), one for key light brightness (light coming from the left side of the screen) and the other for fill light brightness (light coming from the right side of the screen). The tick on each slider could be moved continuously in the range between $12 \%$ and $110 \%$, set to match the range of Tool $\mathrm{B}$. The numeric values of the selected values were not shown on the sliders for either tool. The $100 \%$ was set to be the maximum possible intensity before highlight clipping occurred. All lights were white with the white-point temperature and peak luminanace of the experiment monitor, Dell UP2713H, calibrated to $100 \%$ sRGB color gamut, $6500 \mathrm{k}$, and $80 \mathrm{~cd} / \mathrm{m}^{2}$ brightness.

Tool B also had two control sliders (Figure 1, right), one for the overall brightness and the other for the shadow amount, defined by the key-to-fill ratio (KTFR). The KTFR is the proportion of the key light brightness to the fill light brightness that project on to the character face. High KTFR means there is less illumination, and hence, more shadow on the fill side of the face. The sliders of Tool B could only select a finite combinations of 4 levels of overall brightness $(100 \%, 50 \%, 25 \%$ and $12.5 \%$ and 4 levels of KTFR (1:1, 2:1, $4: 1$ and 8:1). For each pair of brightness and KTFR, the intensity of key and fill light in the scene were adjusted automatically according to an approximation of the parametric model described by Wisessing et al. [2019]. Note that the model maintains consistent iso-brightness level for different KTFRs, and hence the individual light intensity can vary from $12 \%$ to $110 \%$. Please see the mentioned paper for computation details.

\section{EXPERIMENT}

The experiment was divided into two blocks, one for each of the lighting tools, A and B, and we counter-balanced the ordering of the blocks. In each block, the experiment started with a training session, in which the participant was explained how each slider modified the lighting in the scene. The participant took as much time as needed before continuing to the actual experiment. After the training, the participant was asked to complete three tasks (see below).

For the first two tasks, we chose the Mery character animated to portray happiness and sadness, as these two emotions were studied extensively previously. For the final task, we used the neutral animation. Each animation clip lasted 2-3 seconds.

Sixteen participants took part in the experiments (7F, 9M, aged 18-37, avg. 28), all with normal or corrected to normal vision, and recruited primarily via university student and staff mailing lists (from the same population as the previous study by Wisessing et al. [2020]). They had different degrees of experience in CG content creation and were naïve to the purpose of the experiment. A $€ 5$ voucher was rewarded to each volunteer for taking part. The entire experiment was conducted in a completely dark room with no interference of outside light.

The participant instructions were outlined on a piece of paper that was explained clearly to the participant who was also given a copy before the experiment started. In each block, after the training, participants always started with either the Intensity or Appeal task, counter-balanced, each consisting of two trials, happiness and sadness, also counter-balanced. Next, the participant completed the Speed \& Accuracy task and a usability questionnaire. There were 26 trials in total (13 for each block), and participants were allowed to take a short break before the beginning of each trial.

\subsection{Intensity Task}

The participant was instructed to "please light the character for the happiest appearance" for the happy animation, and to light the character for the saddest appearance for the sad depiction. The instruction was also displayed on the top of the screen. The participant was also advised to take as much time as needed. The final selected slider values were recorded for each of the two trials.

\subsection{Appeal Task}

The participant was instructed to "please light the character for the most appealing appearance". The instruction was also displayed on the top of the screen. They were also advised to take as much time as needed and explained the definition of appeal as "If a character is appealing then you would be captivated by a movie with that character in the leading role, and would like to watch more of them." [Kokkinara and McDonnell 2015]. As before, the final selected slider values were recorded for each of the two trials.

\subsection{Speed \& Accuracy Task}

The participant was instructed to "please match your lighting to the target image as close and as quick as possible." The instruction was also displayed on the top of the screen. There were 9 trials in total for this task. The 9 targets, 3 levels of brightness $(100 \%, 50 \%$ and $25 \%$ ) times 3 levels of KTFR (1:1, 2:1 and 4:1), were regular samples of the character lighting perceptual space detailed in [Wisessing et al. 2020]. The slider values and the time taken to complete each trial were recorded. This task simulated the real-word use case of 
a lighting artist performing a set of lighting assignments under a time-constraint.

\subsection{Usability Questionnaire}

After completing the three tasks, the participant was asked to answer a short perceived usability questionnaire designed based on the Usability Metric for User Experience (UMUX) [Finstad 2010]. UMUX was designed to replace the common 10-item, five-point Likert-scale System Usability Scale (SUS) with just four seven-point questions written in less ambiguous language that still conforms to the ISO 9241-11 (1998) definition of usability (overall usability, effectiveness, efficiency and satisfaction), and has been proven to be reliable and highly correlated to SUS [Berkman and Karahoca 2016]. Each question was rated on a seven-point scale from strongly disagree to strongly agree (Figure 4).

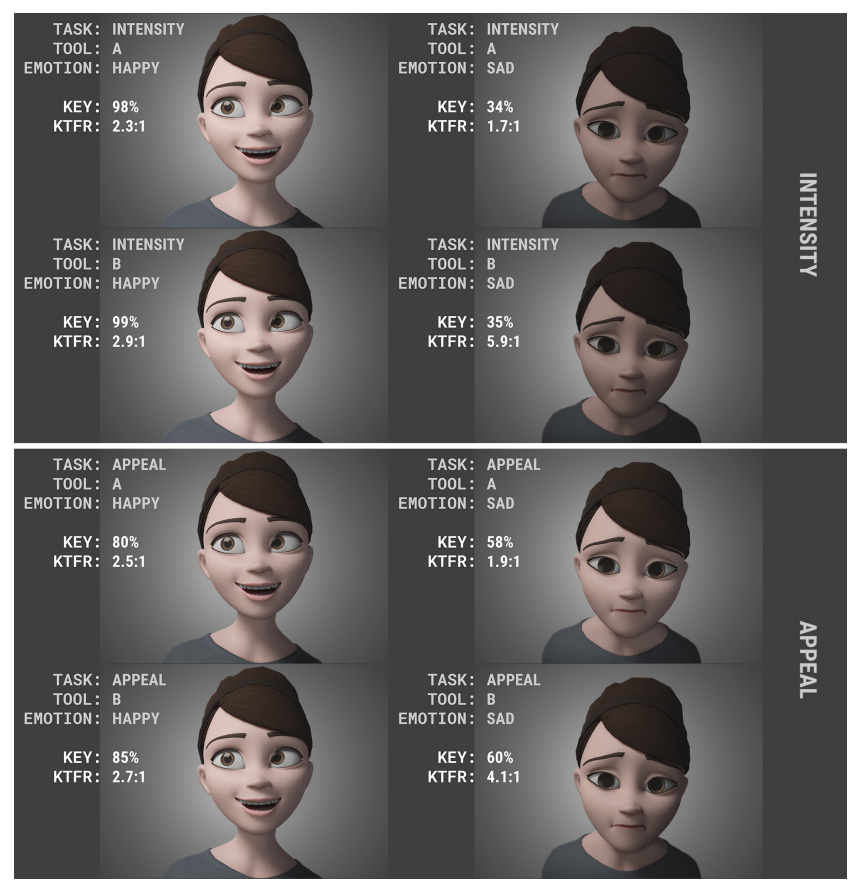

Figure 2: Screenshots from our real-time system showing the average selected key and KTFR values for the Intensity (top) and Appeal (bottom) tasks, for Tool A and B under happiness and (left) sadness (right) emotion.

\section{RESULTS}

For each task, the chosen values of lighting parameters from all participants were analyzed together using a two-way repeatedmeasures ANOVA with the within-group factors of emotion (happiness, sadness) and tool (A, B). In order to be able to compare results across the tools, we generated KTFR values using the key and fill slider values for Tool A. We present the analysis in Figure 3, and only discuss significant results in the text. We ran post-hoc analysis using Tukey's HSD tests throughout.

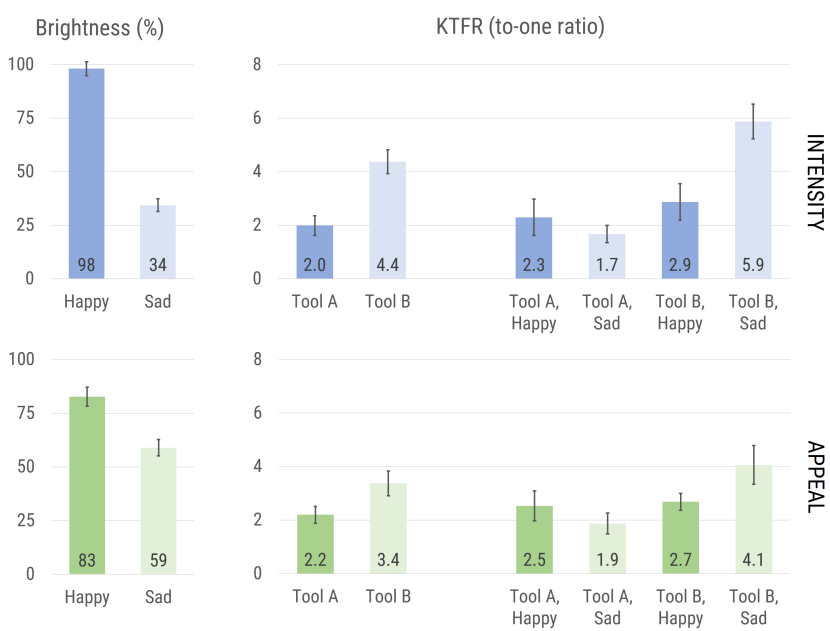

Figure 3: Main effects and interactions of intensity (top) and appeal (bottom) task.

\subsection{Intensity}

Participants were asked to light the character for the most intense appearance and took on average 37.40s to complete each trial. For brightness, we found a main effect of emotion $(F(1,15)=$ $143.89, p<0.0001)$ where happiness $(98 \%)$ was illuminated brighter than sadness (34\%), as expected. A main effect of tool was not found, indicating that participants did not differ in their brightness selections using either tool.

For KTFR, there was no main effect of emotion, indicating that the same KTFR levels were considered intense for happiness and sadness equally. A main effect of tool $(F(1,15)=17.03, p<0.001)$ showed us that participants used higher KTFR with Tool B (4.4:1) than Tool A (2:1), perhaps due to the discrete allowed values of Tool $B$. However, the mean KTFR from Tool B was almost two levels higher than the mean from Tool A indicating that different levels of discretization are needed for a conclusive explanation.

An interaction between tool and emotion $(F(1,15)=17.22, p<$ $0.0003)$ for KTFR showed that sad Mery was lit at higher ratio with Tool B (5.9:1) than Tool A (1.7:1). Also, when using Tool B, participants chose higher KTFR for sad (5.9:1) than for happy (2.9:1) $(p<0.002)$. See Figure 2, top for a visualization of the average chosen values for Tool A and Tool B, and Appendix, Figure 5 for the individual data points selected by participants.

\subsection{Appeal}

Participants were asked to light the character for the most appealing appearance and took on average $35.65 \mathrm{~s}$ to complete each trial. For brightness, the analysis showed a main effect of emotion $(F(1,15)=17.27, p<0.001)$ where happiness $(83 \%)$ was lit brighter than sadness (59\%). For KTFR, a main effect of tool $(F(1,15)=4.61, p<0.049)$ showed that participants chose higher KTFR with Tool B (3.4:1) than Tool A (2.2:1). When asked to light the sad character, participants selected higher KTFR with Tool B (3.4:1) than Tool A (2.2:1) (as indicated by the tool $\mathrm{x}$ emotion interaction $(F(1,15)=7.02, p<0.019))$. There was no main effect of emotion 
on the choice of KTFR, indicating that appealing KTFRs were the same for sad and happy. See Figure 2, bottom for a visualization of the average chosen values for Tool A and Tool B, and Appendix, Figure 5 for the individual data points selected by participants.

\subsection{Speed}

Participants were asked to match the lighting of a character to provided targets. The ANOVA showed a main effect of tool $(F(1,15)=$ $11.21, p<0.005)$ where, on average, that participants completed the task significantly faster when they used Tool B (15.47s) than they did with Tool A (18.73s), indicating that the perceptually-based tool indeed reduced the experiment time for participants as they were not exploring parameters that had no perceptible difference, like in Tool A.

\subsection{Accuracy}

We also tested the accuracy of both tools by mapping the results into the perceptual space using the parametric model from [Wisessing et al. 2019] and then measuring the perceived dissimilarity distances between the results and targets. The ANOVA analysis showed the accuracy of Tool A and B are not significantly different.

\subsection{Usability}

The ANOVA Analysis of the perceived usability questionnaire showed no significant differences of perceived effectiveness, satisfaction, efficiency and the over experience between Tool A and Tool B. In general, effectiveness was rated high, satisfaction was good, overall ease of use was high, and the efficiency was better than average (Figure 4).

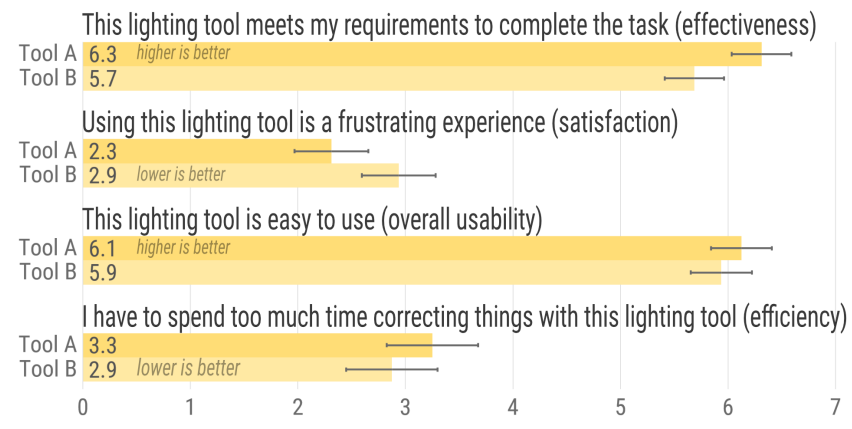

Figure 4: Averaged ratings of the usability questionnaire.

\section{VALIDATION}

Previous work has discussed a need to navigate through the perceptual space of appeal quicker and more effectively [Zell et al. 2019]. This study served as a bridge between the recent findings from the constant stimuli to the new paradigm of interactive experiment design utilizing the modern graphics hardware and game engines. Here, we compare our results to the results of Wisessing et al. [2020]'s extensive set of experiments using the method of constant stimuli to determine if we arrive at similar conclusions.

\subsection{Brightness}

In the previous study, for happiness, brightness level $100 \%$ was rated more intense and more appealing than $25 \%$. Our results here agree as participants selected high levels of brightness for both appeal (98-99\%) and intensity (80-85\%). For sadness, previous work found an effect of brightness on intensity, where the emotion was rated more intense at $100 \%$ than at $25 \%$. Our result here agrees, and we additionally hone-in on the optimal brightness level for intensity of sadness at $34 \%$. In the previous study, there was no difference between ratings of appeal for the $100 \%$ or $25 \%$ brightness levels. Our result here complements that finding and also provides the optimum brightness for appeal of sadness of $58-60 \%$.

\subsection{KTFR}

In terms of shadow amount, the previous study did not find any conclusive evidence that indicated the effects of KTFR on the intensity and appeal of happiness and sadness which contradicts the artistic practice of using shadow to intensify the drama. However, our result gave us ranges of KTFR that the audience preferred for emotional and appealing characters. This could be influenced by their past experience and the debate on whether top-down or bottom-up processing is better for lighting perception is worth investigating in future work.

\subsection{Time}

Lastly, time taken to explore brightness and KTFR parameters was 36-37 seconds per trial for our method-of-adjustment experiment, and 35-40 seconds per trial for Wisessing et al. [2020]'s method-ofconstant-stimuli experiments. Although the speed improvement seems negligible, note that we instructed our participants to take as much time as they needed. Furthermore, if the previous work matched their thresholds to our Tool B, their experiment would have taken more than 60 minutes to complete, rendering the results less reliable due to fatigue. With the same amount of time, our experiments could explore a broader range of parameters and produced results with exact thresholds.

\section{CONCLUSION}

In this paper, we presented a real-time lighting tool that could be used in method-of-adjustment perceptual experiments investigating lighting parameters. We evaluated the tool by allowing users to interactively alter the light brightness and shadow on animated cartoon characters. We included separate tasks for lighting for speed and accuracy, lighting for appeal, and lighting to improve emotional intensity. Our results were compared to previous work that used a method-of-constant-stimuli with a Likert-scale response, and we validated their findings, while our experiment identified the exact thresholds for appeal and intensity. We expect that our method of adjustment will speed-up investigation of experiments with large ranges of parameters. However, one limitation of method of adjustment is that the decision task gets more difficult as the number of dimensions increase, so we will investigate it's usability with 3 or more sliders in future work. In terms of usability, participants found the tools easy to use and met their requirements to complete the tasks, which indicates that this method could prove useful for future analysis of a wider range of lighting parameters, allowing 
researchers to analyze the perception of virtual characters more rapidly.

\section{ACKNOWLEDGMENTS}

This research was funded by Science Foundation Ireland under the ADAPT Centre for Digital Content Technology (Grant 13/RC/2106) and the Game Face (Grant 13/CDA/2135) project. We thank the Mery Project for the character model and rig.

\section{REFERENCES}

Kimberly A. Barchard, Kelly E. Grob, and Matthew J. Roe. 2017. Is sadness blue? The problem of using figurative language for emotions on psychological tests. Behavior Research Methods 49, 2 (2017), 443-456.

Mehmet Ilker Berkman and Dilek Karahoca. 2016. Re-assessing the usability metric for user experience (UMUX) scale. Journal of Usability Studies 11, 3 (2016), 89-109.

Douglas W Cunningham and Christian Wallraven. 2011. Experimental design: From user studies to psychophysics. AK Peters/CRC Press.

Gustav Theodor Fechner, Davis H Howes, and Edwin Garrigues Boring. 1966. Elements of psychophysics.

Kraig Finstad. 2010. The usability metric for user experience. Interacting with Computers 22, 5 (2010), 323-327.

Reuben Fleming, Betty J. Mohler, Javier Romero, Michael J. Black, and Martin Breidt. 2016. Appealing Female Avatars from 3D Body Scans: Perceptual Effects of Stylization. In Int. Conf. on Computer Graphics Theory and Applications (GRAPP).

Simon Grondin. 2016. Psychology of perception. Springer.

Elena Kokkinara and Rachel McDonnell. 2015. Animation realism affects perceived character appeal of a self-virtual face. In Proceedings of the 8th ACM SIGGRAPH Conference on Motion in Games. ACM, 221-226.

Yuki Koyama, Issei Sato, Daisuke Sakamoto, and Takeo Igarashi. 2017. Sequential line search for efficient visual design optimization by crowds. ACM Transactions on Graphics (TOG) 36, 4 (2017), 1-11.

Mark J Landau, Brian P Meier, and Lucas A Keefer. 2010. A metaphor-enriched social cognition. Psychological bulletin 136, 6 (2010), 1045.

Ross Lowell. 1992. Matters of light \& depth: creating memorable images for video, film $\&$ stills through lighting. Broad Street Books.

Karl F. MacDorman, Robert D. Green, Chin-Chang Ho, and Clinton T. Koch. 2009. Too real for comfort? Uncanny responses to computer generated faces. Computers in Human Behavior 25, 3 (2009), 695-710.

Rachel McDonnell, Martin Breidt, and Heinrich Buelthoff. 2012. Render me Real \{I\}nvestigating the Effect of Render Style on the Perception of Animated Virtual Humans. ACM Transactions on Graphics 31, 4 (2012), 91:1--91:11.

Brian P Meier and Michael D Robinson. 2005. The metaphorical representation of affect. Metaphor and symbol 20, 4 (2005), 239-257.

Brian P Meier, Michael D Robinson, and Gerald L Clore. 2004. Why good guys wear white: Automatic inferences about stimulus valence based on brightness. Psychological science 15,2 (2004), 82-87.

Frank Thomas and Ollie Johnston. 1995. The illusion of life: Disney animation. Hyperion New York.

Matias Volante, Sabarish V Babu, Himanshu Chaturvedi, Nathan Newsome, Elham Ebrahimi, Tania Roy, Shaundra B Daily, and Tracy Fasolino. 2016. Effects of virtual human appearance fidelity on emotion contagion in affective inter-personal simulations. IEEE Transaction on Visualization and Computer Graphics 22, 4 (2016), 1326-1335.

Christian Wallraven, Heinrich H. Bülthoff, Douglas W. Cunningham, Jan Fischer, and Dirk Bartz. 2007. Evaluation of real-world and computer-generated stylized facial expressions. ACM Transactions on Applied Perception 4, 3, Article 16 (2007).

Pisut Wisessing, John Dingliana, and Rachel McDonnell. 2016. Perception of Lighting and Shading for Animated Virtual Characters. In Proc. of ACM Symp. of Applied Perception (SAP). 25-29.

Pisut Wisessing, Katja Zibrek, Douglas W Cunningham, John Dingliana, and Rachel McDonnell. 2020. Enlighten Me: Importance of Brightness and Shadow for Character Emotion and Appeal. ACM Transactions on Graphics (TOG) 39, 3 (2020),
$1-12$.

Pisut Wisessing, Katja Zibrek, Douglas W. Cunningham, and Rachel Mcdonnell. 2019. A psychophysical model to control the brightness and key-to-fill ratio in CG cartoon character lighting. In ACM Symposium on Applied Perception 2019. 1-7.

Alison Jing Xu and Aparna A Labroo. 2014. Incandescent affect: Turning on the hot emotional system with bright light. Journal of Consumer Psychology 24, 2 (2014), 207-216.

Akiko Yoshida, Rafał Mantiuk, Karol Myszkowski, and Hans-Peter Seidel. 2006. Analysis of reproducing real-world appearance on displays of varying dynamic range. In Computer Graphics Forum, Vol. 25. Wiley Online Library, 415-426.

Eduard Zell, Carlos Aliaga, Adrian Jarabo, Katja Zibrek, Diego Gutierrez, Rachel McDonnell, and Mario Botsch. 2015. To Stylize or Not to Stylize?: The Effect of Shape and Material Stylization on the Perception of Computer-generated Faces. ACM Trans. Graph. 34, 6 (oct 2015), 184:1--184:12.

Eduard Zell, Katja Zibrek, and Rachel McDonnell. 2019. Perception of virtual characters. In ACM SIGGRAPH 2019 Courses. 1-17.

\section{APPENDIX}
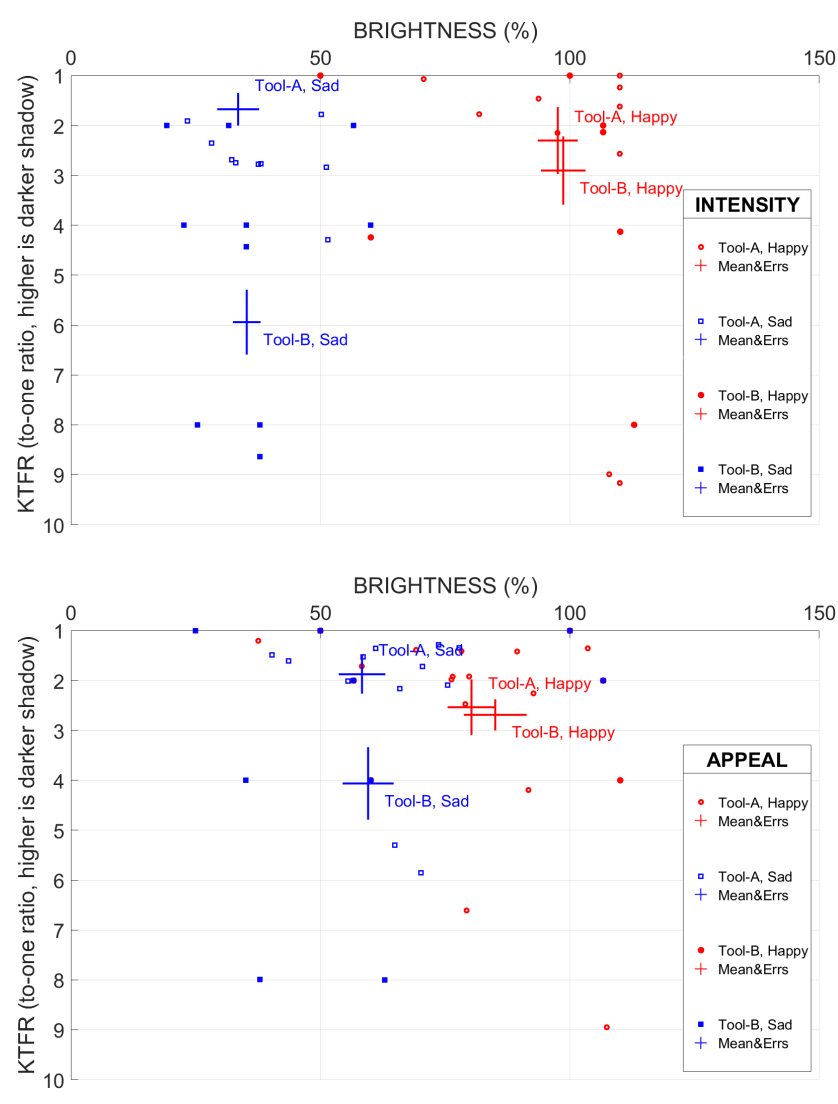

Figure 5: Individual data points of the intensity and appeal tasks selected by the participants. 